\title{
Microalbuminuria In Children And Adolescents With Type 1 Diabetes Mellitus: Predictive Factors
}

Authors: Joana Menezes Nunes ${ }^{1,2,3}$, Ricardo Monteiro4, Daniela Amaral ${ }^{5}$, Rosa Pina ${ }^{5}$, Lurdes Lopes ${ }^{5}$, Catarina Limbert5,6

1 - Endocrinology, Diabetes and Metabolism Department, Centro Hospitalar São João, Porto; 2 - Faculty of Medicine, University of Porto; 3 Instituto de Investigação e Inovação em Saúde, University of Porto; 4 - Pediatric Department, Centro Hospitalar Leiria Pombal, Leiria, Portugal; 5 -

Pediatric Endocrinology Department, Hospital D. Estefânia, Lisboa; 6 - Faculty of Medicine, Lisboa University. Portugal

\section{OBJECTIVES}

Type 1 diabetes mellitus (T1DM) is increasing worldwide and is the most common chronic endocrine disease in children.

Microalbuminuria (MA) is usually the first microvascular complication of T1DM. According to the latest consensus report of the International Society for Pediatric and Adolescent Diabetes (ISPAD), it is recommended to screen annually for microvascular complications from age 10-years or at the onset of puberty if earlier and after two to five years' diabetes duration.

We aimed to identify MA frequency, time of occurrence and related risk factors and if there is a relationship between nephropathy and obesity in our cohort of T1DM.

\section{METHODS}

Clinical reports of 201 children with T1DM diagnosed at least $>1$ year ago followed in our institution were retrospectively analysed.

Statistical analysis was performed with SPSS ${ }^{\circ}$ version 21 for Windows ${ }^{\circledR}$.

Results are expressed in frequencies and means \pm standard deviations and statistical significance was considered as $p$ value $<0.05$.

MA was defined as ACR $>30 \mathrm{mg} / \mathrm{g}$ in at least two consecutive spot urine samples collected in the same year.

\section{RESULTS}

\section{PATIENTS' CHARACTERISTICS}

\begin{tabular}{|c|c|c|c|c|}
\hline & TOTAL & FEMALE & MALE & P VALUE \\
\hline Number (nr) & 201 & 102 & 99 & NS \\
\hline Actual age (y) & $12.4 \pm 3.9$ & $12.6 \pm 3.8$ & $12.2 \pm 4.0$ & NS \\
\hline $\begin{array}{c}\text { Age at T1DM } \\
\text { diagnosis (y) } \\
<10(\mathrm{nr}) \\
10-15(\mathrm{nr}) \\
>15(\mathrm{nr})\end{array}$ & $\begin{array}{c}8.1 \pm 3.7 \\
147 \\
47 \\
7\end{array}$ & $\begin{array}{c}7.9 \pm 3.5 \\
77 \\
23 \\
2\end{array}$ & $\begin{array}{c}8.3 \pm 3.8 \\
70 \\
24 \\
5\end{array}$ & NS \\
\hline T1DM duration (y) & $3.8 \pm 3.6$ & $4.3 \pm 3.6$ & $3.4 \pm 3.5$ & NS \\
\hline HbA1c (\%) & $9.0 \pm 1.8$ & $9.0 \pm 1.9$ & $8.9 \pm 1.7$ & NS \\
\hline BMI (Kg/m²) & $20.6 \pm 4.3$ & $21.4 \pm 4.6$ & $19.7 \pm 3.7$ & 0.004 \\
\hline No Hypertension & $201(100 \%)$ & - & - & - \\
\hline Dyslipidemia & $84(41.8 \%)$ & $50(49.0 \%)$ & $34(34.3 \%)$ & 0.045 \\
\hline MA & 17 & $10(58.8 \%)$ & $7(41.2 \%)$ & NS \\
\hline $\begin{array}{l}\text { TC (mg/dL) } \\
<170(\mathrm{nr}) \\
170-199(\mathrm{nr}) \\
\geq 200(\mathrm{nr})\end{array}$ & $\begin{array}{c}161.2 \pm 34.8 \\
116 \\
41 \\
18\end{array}$ & $\begin{array}{c}168.3 \pm 40.3 \\
54 \\
25 \\
13\end{array}$ & $\begin{array}{c}153.4 \pm 25.6 \\
62 \\
16 \\
5\end{array}$ & 0.04 \\
\hline $\begin{array}{l}\text { LDL-c (mg/dL) } \\
\quad<100(\mathrm{nr}) \\
\quad 100-129(\mathrm{nr}) \\
\geq 130(\mathrm{nr})\end{array}$ & $\begin{array}{c}112.4 \pm 26.3 \\
92 \\
48 \\
36\end{array}$ & $\begin{array}{c}117.0 \pm 28.0 \\
42 \\
24 \\
26 \\
\end{array}$ & $\begin{array}{c}107.3 \pm 23.4 \\
50 \\
24 \\
10\end{array}$ & 0.01 \\
\hline $\begin{array}{l}\text { HDL-c (mg/dL) } \\
\quad<35(\mathrm{nr}) \\
35-44(\mathrm{nr}) \\
\geq 45(\mathrm{nr})\end{array}$ & $\begin{array}{c}53.2 \pm 12.1 \\
9 \\
35 \\
132 \\
\end{array}$ & $\begin{array}{c}54.2 \pm 13.7 \\
6 \\
15 \\
71 \\
\end{array}$ & $\begin{array}{c}52.2 \pm 10.1 \\
3 \\
20 \\
61 \\
\end{array}$ & NS \\
\hline $\begin{array}{l}\text { TG (mg/dL) } \\
\quad<100(\mathrm{nr}) \\
100-150(\mathrm{nr}) \\
\geq 150(\mathrm{nr})\end{array}$ & $\begin{array}{c}83.9 \pm \mathbf{5 8 . 0} \\
138 \\
28 \\
10\end{array}$ & $\begin{array}{c}94.5 \pm 74.0 \\
67 \\
17 \\
8\end{array}$ & $\begin{array}{c}72.2 \pm 29.0 \\
71 \\
11 \\
2\end{array}$ & 0.009 \\
\hline
\end{tabular}

\section{PATIENTS WITH MA - CHARACTERISTICS}

CHARACTERISTICS

Number (nr)

Gender

Age at MA diagnosis (y)

$\Delta t$ to MA after T1DM onset (y)

MA present within the first $5 \mathrm{y}$ of T1DM duration

MA present within the first $2 y$ of T1DM duration

Age at T1DM diagnosis $(y)$

$<10(\mathrm{nr})$

10-15 (nr)

$>15(\mathrm{nr})$

Hypertension
RESULTS

17 (8.5\%)

Female: 10

Male: 7

$11.1 \pm 3.3[6.4 ; 14.5]$

$5.5 \pm 3.8[0.75 ; 14.0]$

9 children $(52.9 \%)$

5 during puberty; 4 befor puberty

5 children $(29.4 \%)$

3 during puberty; 2 before puberty

$\mathbf{8 . 4} \pm \mathbf{3 . 7}$

$12(70.6 \%)$

$5(29.4 \%)$

$0(0 \%)$

$0(0 \%)$

\section{LOGISTIC REGRESSION ANALYSIS}

\begin{tabular}{|c|c|c|c|}
\hline VARIABLE & OR & P VALUE & Cl $95 \%$ \\
\hline Actual age & $1.09^{*}$ & 0.037 & {$[1.02 ; 1.17]$} \\
\hline Age at diagnosis & 0.98 & NS & {$[0.85 ; 1.12]$} \\
\hline T1DM duration & 1.38 & 0.005 & {$[1.11 ; 1.59]$} \\
\hline Gender & 1.34 & NS & {$[0.74 ; 1.11]$} \\
\hline BMI & 1.13 & 0.026 & {$[1.04 ; 1.26]$} \\
\hline HbA1c & 1.88 & 0.021 & {$[1.45 ; 2.19]$} \\
\hline TC & 1.26 & 0.033 & {$[1.07 ; 1.44]$} \\
\hline LDL-c & 1.44 & 0.042 & {$[1.22 ; 1.63]$} \\
\hline HDL-c & 1.03 & NS & {$[0.95 ; 1.09]$} \\
\hline TG & 1.63 & 0.006 & {$[1.31 ; 1.78]$} \\
\hline
\end{tabular}

* Adjusted for T1DM duration

Abbreviations: TC $=$ total cholesterol; $T G=$ triglycerides; $N S=$ non-significant

\section{CONCLUSIONS}

In children, nephropathy can occur soon after T1DM onset.

Our results have provided important data on prevalence, predictive factors and screening of nephropathy in a young population of T1DM patients

Besides the well-described risk factors for MA (namely poor metabolic control and longer diabetes duration), we also found obesity and dyslipidemia to increase the risk of nephropathy.

According to our results, we suggest yearly screening of MA after T1DM onset and early treatment of dyslipidemia and obesity.

\section{REFERENCES}

ISPAD Clinical Practice Consensus Guidelines 2014
Compendium; Microvascular and macrovascular
complications in children and adolescentes. Pediatric
Diabetes 2014: 15 (Suppl. 20): $257-269$
Cho YH et al. Microvascular complications assessment
in adolescents with 2- to 5-yr duration of type 1 diabetes
from 1990 to 2006. Pediatr Diabetes, 2011; 12: $682-689$.
SM Marshall: Natural history and clinical characteristics
of CKD in type 1 and type 2 diabetes mellitus. Adv
Chronic Kidney Dis. 2014; $21(3): 267-272$
Sweiss N, Sharma K: Adiponectin effects on the kidney.
Best Pract Res Clin Endocrinol Metab 2014; 28(19): 71 -
79

SPAD Clinical Practice Consensus Guidelines 2014 Compendium, Microvascular and macrovascular Diabetes 2014: 15(Suppl. 20): 257-269 Ch al. Microvascular complications assessment from 1990 to 2006. Pediatr Diabetes, 2011; 12: 682-689. SM Marshall: Natural history and clinical characteristics Chronic Kidney Dis. 2014; 21(3): 267-272 